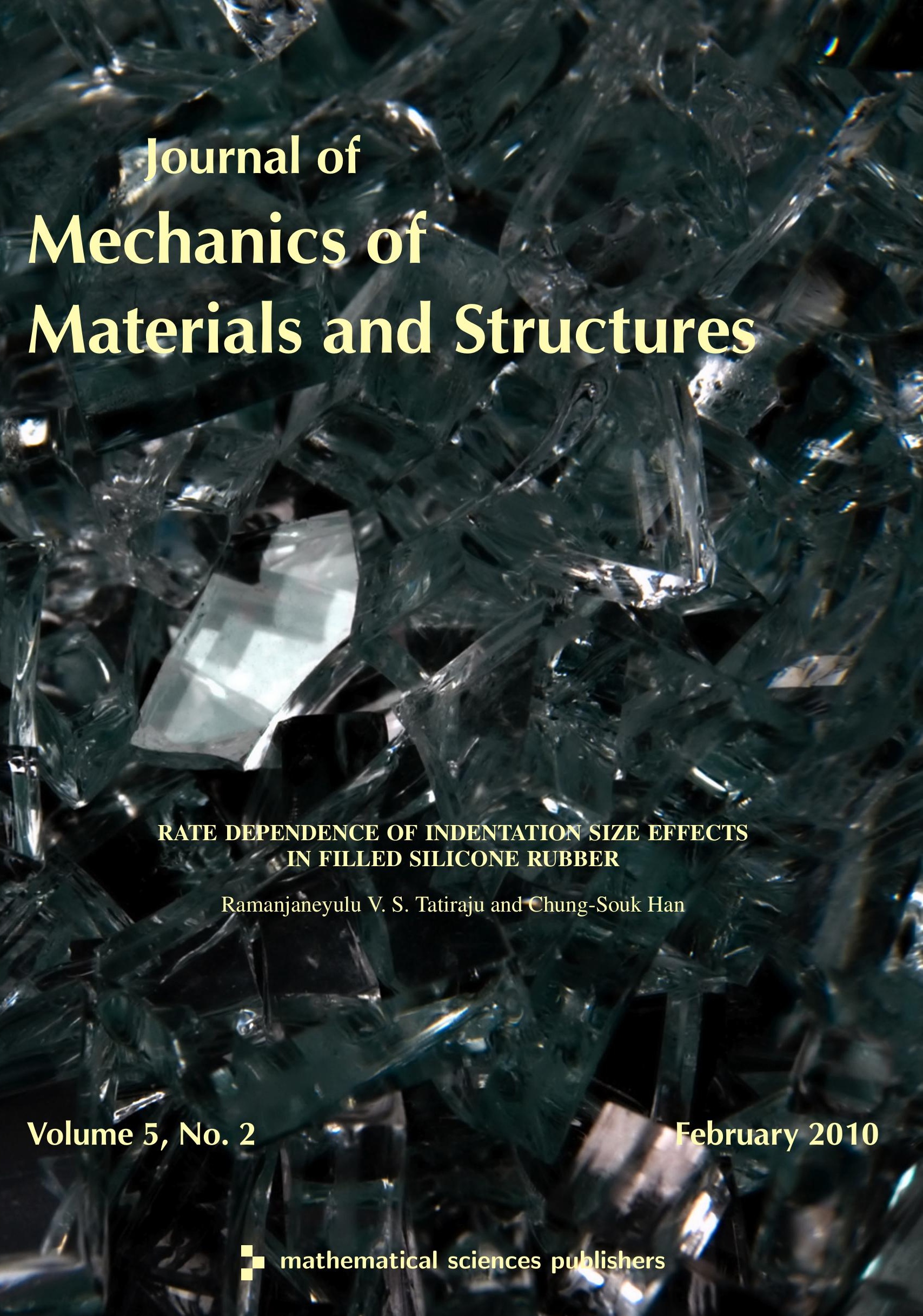




\title{
RATE DEPENDENCE OF INDENTATION SIZE EFFECTS IN FILLED SILICONE RUBBER
}

\author{
RAMANJANEyUlu V. S. TATIRAJU AND Chung-SOUK HaN
}

\begin{abstract}
Indentation experiments at the nanometer and micrometer range reported in the literature have shown that the deformation of polymers is - similarly to metals - size-dependent. In addition its size dependence, the deformation behavior of polymers is also known to be rate-dependent. Here silicone rubber is considered. In order to characterize the relation between size and rate-dependent deformation indentation tests of silicone at indentation depths of between 30 and 300 microns and loading times of between 1 and 1000 seconds are performed. In these experiments the hardness and dissipation increased with decreasing loading time and decreasing indentation depth. These experimental results are analyzed with a recently suggested indentation model which incorporates a Frank energy-related nonlocal deformation work. It is found that the indentation model is in good agreement with the experimental data. Evaluation of the experimental data indicates that the rate effects are mostly related to the nonlocal, size-dependent deformation.
\end{abstract}

\section{Introduction}

The indentation size effect manifests itself in increasing hardness at decreasing indentation depths and is fairly well investigated for metals [Ma and Clarke 1995; Nix and Gao 1998; Han et al. 2007a]. Such elevated hardnesses at small indentation depths have also been observed in polymers [Briscoe et al. 1998; Chong and Lam 1999; Tjernlund et al. 2004; Lim and Chaudhri 2006; Han and Nikolov 2007] but, in comparison to metals, for polymers these effects are not well understood. An understanding of size-dependent deformation behavior is important not only for small components in MEMS/NEMS (micro/nano-electromechanical systems), but also for the development of advanced material systems and composite materials with small material phases and other applications such as sealants, gaskets [Tan et al. 2007], and adhesives [Han et al. 2007b]. As many biological materials can be viewed as polymers, the relevance of size effects in polymers extends also to these materials [Yang et al. 2002].

Rate effects in indentation testing have been studied in [Sargent and Ashby 1992; Larsson and Carlsson 1998; Ebenstein and Wahl 2006; Tweedie and Van Vliet 2006], for instance, but without considering size effects. The effects of cross-linking and adhesion on the determination of the elasticity modulus of polydimethylsiloxane elastomers have been studied in [Carrillo et al. 2005]. A phenomenological constitutive model incorporating both rate and size effects has been suggested in [Gudmundson 2006], for instance. The elasticity modulus has also been determined to vary with the indentation depth [McFarland and Colton 2005; Tweedie et al. 2007]; this may be seen as a indication that a change in the dominant forces or mechanisms may be present at small indentation depths.

Keywords: indentation, hardness, polymeric material, viscoelasticity, size dependent deformation. 
In [Han and Nikolov 2007] indentation experiments in the literature have been analyzed, and it has been found that the indentation size effect strongly depends on the polymeric material and its molecular structure [Han 2010]. For instance, Teflon (polytetrafluoroethylene) [Li and Bhushan 2000] does not seem to show any indentation size effects, while epoxy and polycarbonate show significant indentation size effects at indentation depths from $100 \mathrm{~nm}$ up to several microns [Chong and Lam 1999].

Assuming the rate of deformation does not alter the basic characteristics, these experimental results were evaluated with a hardness model suggested in [Han and Nikolov 2007]. The model and experimental results were found to be in good agreement for indentation depths above some $200 \mathrm{~nm}$. The model is based on an elastoplastic extension of the rotation gradient model suggested in [Nikolov et al. 2007], which relates the length scale component of the deformation work to the Frank energy known from liquid crystal polymers. For indentation depths smaller than $200 \mathrm{~nm}$ surface effects may be present which are not represented in the hardness model. In metals such surface effects may include the effect of the curvature of the indenter tip [Qu et al. 2004], different deformation mechanisms close to free surfaces [Han et al. 2006], and surface roughness effects [Zhang and Xu 2002; Zhang et al. 2004].

Among the different polymers tested for size-dependent deformation, silicone rubber appears to exhibit astonishing behavior. While at the macroscale silicone rubber is very soft, at indentation depths of under one micron it exhibits higher hardness than many macroscopically harder polymers [Zhang and Xu 2002; $\mathrm{Xu}$ and Zhang 2004; Han and Nikolov 2007]. Here we investigate the rate dependence of the indentation size effect in silicone rubber with indentation tests at indentation depth ranges of approximately 30300 microns and in loading time ranges of 1-1000 seconds. The data for these experiments are analyzed and discussed using the hardness model suggested in [Han and Nikolov 2007].

\section{Experimental setting}

Indentation system. The indentation tests were performed with the commercially available Fischerscope HM2000S indentation system (Fischer Technology Inc., USA). It is a load controlled system for the determination of hardness and other material properties [ISO 14577-1 2002]. The applicable load of the machine ranges between 0.4 and $2000 \mathrm{mN}$ and the loading time between 0.6 and $10^{6}$ seconds. The load and penetration depth resolutions are given by the manufacturer as $\leq 40 \mu \mathrm{N}$ and $\leq 100 \mathrm{pm}$, respectively. Also according to the manufacturer the curvature of the indenter tip is guaranteed to be less than $500 \mathrm{~nm}$, approximately between 200 and $300 \mathrm{~nm}$; only polymeric materials with this indenter tip have been tested.

Silicone rubber material. The ingredients for the silicone rubber sample fabrication were obtained from Gelest, Inc., Morrisville, PA, USA. The silicone materials were produced in two parts, both of them silicone blends. Part A consists of fumed silica-reinforced vinyl-terminated polydimethylsiloxane (Gelest catalog code DMS-V31S15) as a base and a precious metal catalyst for vinyl-addition silicone cure platinum-divinyltetramethyldisiloxane complex in xylene (Gelest SIP 6831.2). Part B consist of vinylterminated polydimethylsiloxanes (Gelest DMS-V31) as vinyl silicone, methylhydrosiloxane-dimethylsiloxane copolymers and a trimethylsiloxy terminated (Gelest HMS-301) cross-linker.

For part A, $84.85 \mathrm{ml}$ of the base was poured into a glass beaker by measuring its mass on a weighing machine; meanwhile, $3 \mathrm{ml}$ of the catalyst was slowly added with a pipette. The compound was then thoroughly mixed with a stirrer for a couple of minutes. For part B, $80 \mathrm{ml}$ of vinyl silicone and $20 \mathrm{ml}$ of cross-linker were thoroughly mixed with a stirrer in another beaker. Parts A and B were poured in a 
proportion of 3:1 into a polypropylene specimen cup. To get rid of any bubbles, the whole mixture was placed in an airtight chamber using a vacuum pump. The aggregate was then poured into a Petri dish (50 $\mathrm{mm}$ in diameter and $9 \mathrm{~mm}$ in depth, Pall Corporation, USA) to avoid damage and straining of the material due to handling. Finally the specimen was allowed to cure for several days at room temperature.

\section{Indentation experiments}

Figure 1 shows the indentation depth $h$ and its projected counterpart $h_{\mathrm{c}}$. Also shown are the elastic, $W_{\mathrm{I}}^{\mathrm{e}}$, and inelastic, $W_{\mathrm{I}}^{\mathrm{p}}$, parts of the total indentation work $W_{\mathrm{I}}=W_{\mathrm{I}}^{\mathrm{e}}+W_{\mathrm{I}}^{\mathrm{p}}$ in the loading and unloading sequence of the load-displacement curve. A Berkovich indenter tip was applied with a characteristic angle of about $65^{\circ} 2^{\prime}$. The nominal contact area is $A_{\mathrm{s}}=c_{\mathrm{B}} h^{2}$ for the Berkovich tip, where $c_{\mathrm{B}}=26.440$ [ISO 14577-1 2002].

The stress and strain fields are highly inhomogeneous during indentation [Dao et al. 2001]. Because of this inhomogeneity, strain fields and strain rates vary strongly. From a classical local continuum mechanics viewpoint, where the constitutive equations are described by the stresses and strains and their rates, these stress and strain fields should be affine to each other with $h$ under certain conditions: first, the indenter tip geometry should be self-affine, that is, the nominal penetrating shape of the indenter tip should scale with the indentation depth, and, second, the strain and strain rate fields should also be affine to the indentation depth at a certain time.

For the self-affine cone geometry of the Berkovich indenter tip used here, the maximum indentation depth $h_{\max }$ is related to the maximal applied force $F_{\max }$ by

$$
h_{\max }^{2}=c_{\mathrm{o}} F_{\max }
$$

where the constant $c_{\mathrm{o}}$ depends on the specific indenter shape, material constants, and loading rise time $t_{\mathrm{R}}$; see [Oyen 2006]. Following the discussion above, the relation (1) should be true irrespective of the maximum applied load if material points experience the same strain rates spatially affine to the maximum indentation depth $h_{\max }$ at all times. In other words, under these conditions the location of a material point with the same strain rate history scales with $h_{\max }$. From a classical continuum mechanical viewpoint this should be fulfilled if the load $F$ goes up from 0 to its maximum $F_{\max }$ linearly in time, as in [Oyen 2006]; in symbols, $F=\left(t / t_{\mathrm{R}}\right) F_{\max }$, where $t_{\mathrm{R}}$ is the load raising time. If the load is raised quadratically then $F=\left(t / t_{\mathrm{R}}\right)^{2} F_{\max }$. If $t_{\mathrm{R}}$ remains the same while $F_{\max }$ changes, the stress fields and the strain and

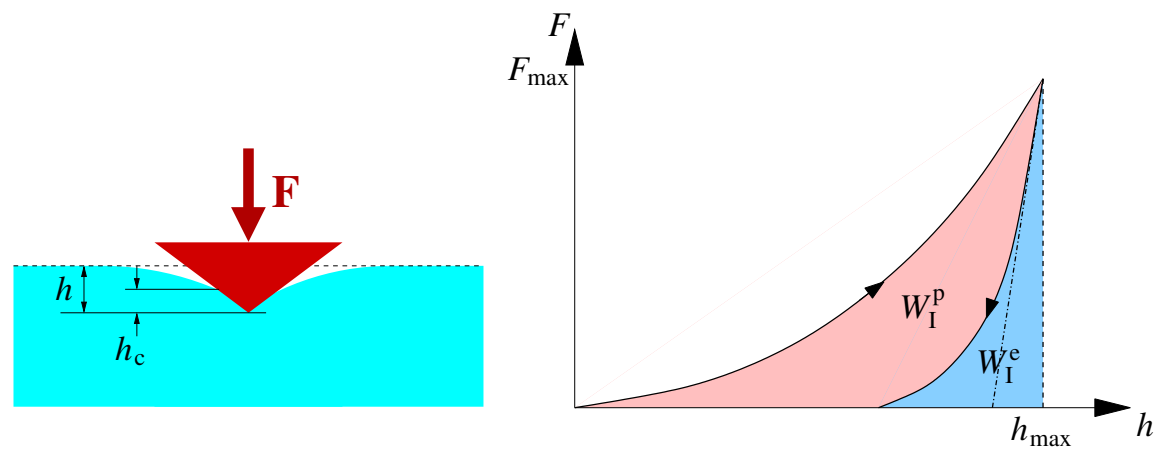

Figure 1. Indentation depths (left) and elastic $W_{\mathrm{I}}^{\mathrm{e}}$ and inelastic $W_{\mathrm{I}}^{\mathrm{p}}$ parts of the total indentation work $W_{\mathrm{I}}$ in a load-displacement diagram (right). 
strain rate fields will be affine with respect to $h_{\max }$. As a direct consequence of (1) the universal hardness $H_{\max }=F_{\max } /\left(c_{\mathrm{B}} h_{\max }^{2}\right)$ is not dependent on $F_{\max }$ or on $h_{\max }$, which is the expected result from a classical continuum mechanical viewpoint in terms of local stresses and strains and their rates.

In order to examine the rate dependence of the silicone rubber various loading times were applied for a given maximum load $F_{\max }$. The load rising and falling time of the load-controlled indentations were chosen to be equal and set to be $t_{\mathrm{R}}=1,10,100$, and 1000 seconds with zero holding time to minimize creep type deformation. The load increased quadratically in time till the maximum prescribed load was reached at $t_{\mathrm{R}}$. The unloading was correspondingly decreased quadratically in the same time interval.

It has been found that the tested silicone material is highly elastic. Typical load-displacement curves with different indentation times are shown in Figure 2, left, including the load-displacement curves of the loading and unloading sequences. The lines in loading and unloading for these loading times can hardly be distinguished. Particularly for the 1000 second test, the indentation depth $h$ at zero load was also practically zero, which indicates that there is little if any plastic deformation. However, to visualize that there is some dissipation, the load displacement curves in loading and unloading for the 1 second test have been redrawn in Figure 2, right.

Essentially no permanent indentation $h$ was observed after the load had been removed, although at small loading times $t_{\mathrm{R}}$ (see Figure 2, right) some indentation depth $h$ is measured at zero applied load. Because there is hardly any remaining indentation depth for the indentations for $t_{\mathrm{R}}=1000 \mathrm{~s}$ it can arguably be assumed that most of the remaining $h$ in Figure 2, right, at the end of the unloading sequence is actually recovered in time. This motivates the use of

$$
W_{\mathrm{I}}=W_{\mathrm{I}}^{\mathrm{e}}+W_{\mathrm{I}}^{\mathrm{v}}
$$

as the decomposition of the indentation work, where $W_{\mathrm{I}}^{\mathrm{v}}$ is the viscous part of the indentation work which, is not recovered.

In Figure 3 the ratio between $W_{\mathrm{I}}^{\mathrm{e}}$ and $W_{\mathrm{I}}$, that is, $\eta^{\mathrm{e}}=W_{\mathrm{I}}^{\mathrm{e}} / W_{\mathrm{I}}$, is illustrated for different indentation depths and loading/unloading times. As depicted therein, with decreasing indentation time the ratio $\eta^{\mathrm{e}}$

$$
F(\mathrm{mN})
$$

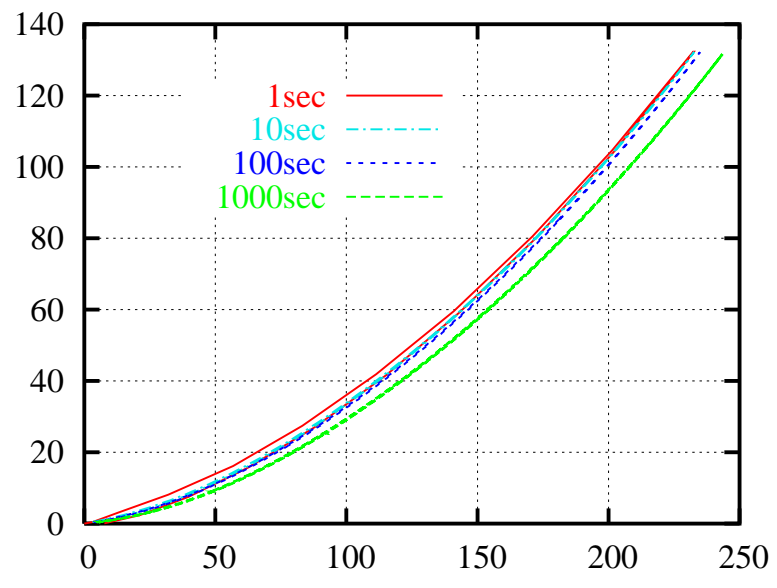

$$
F(\mathrm{mN})
$$

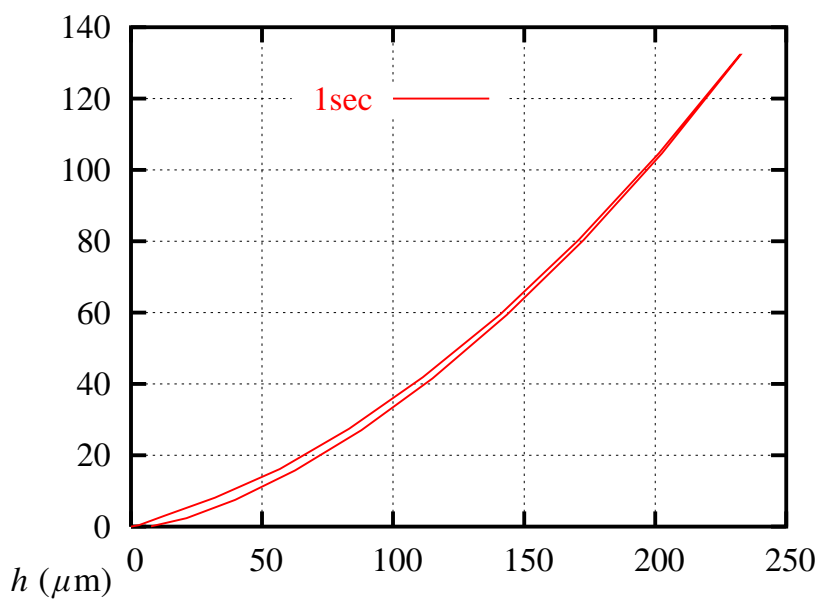

Figure 2. Indentation depth $h$ versus load $F$ at various loading/unloading times (left). The curves for 1 second are repeated on the right. 


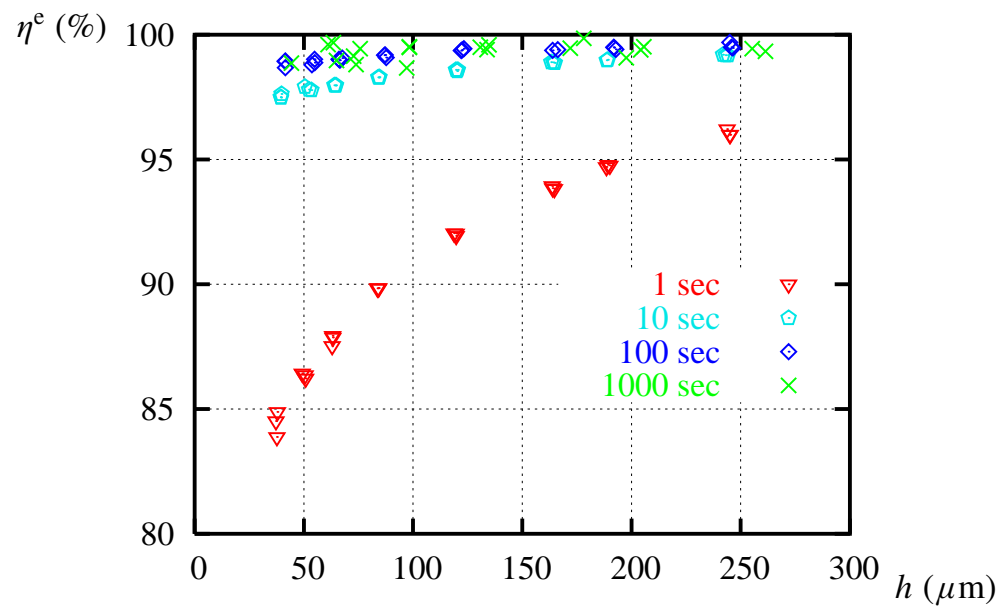

Figure 3. Ratio $\eta^{\mathrm{e}}$ versus indentation depth $h$ for various loading times.

decreases, corresponding to increasing dissipation. Such behavior is well known and widely observed in other material tests such as simple tensile tests [Lemaitre and Chaboche 1990]. The values in Figure 3, however, also show a decrease of $\eta^{\mathrm{e}}$ with decreasing $h$, and therefore the dissipation of the indentation work increases with decreasing $h$. Similar tendencies have also been observed in other polymers [Briscoe et al. 1998; Tatiraju et al. 2008]. The magnitude with which $\eta^{\mathrm{e}}$ decreases is more pronounced at shorter loading times. As the majority of the deformation is elastic and hardly any plastic deformation is present the hardness values characterize elastic rather than plastic deformation.

A material model that is formulated only in local stress and strain related terms would not predict any change in $\eta^{\mathrm{e}}$ with the indentation depth $h$. The changes in $\eta^{\mathrm{e}}$ with $h$ are, however, apparent in Figure 3. The indentation size effect is usually assessed by the relation between hardness and indentation depth. The values of these quantities corresponding to Figure 3 are shown in Figure 4, which plots the universal hardness,

$$
H=F / A_{\mathrm{s}},
$$

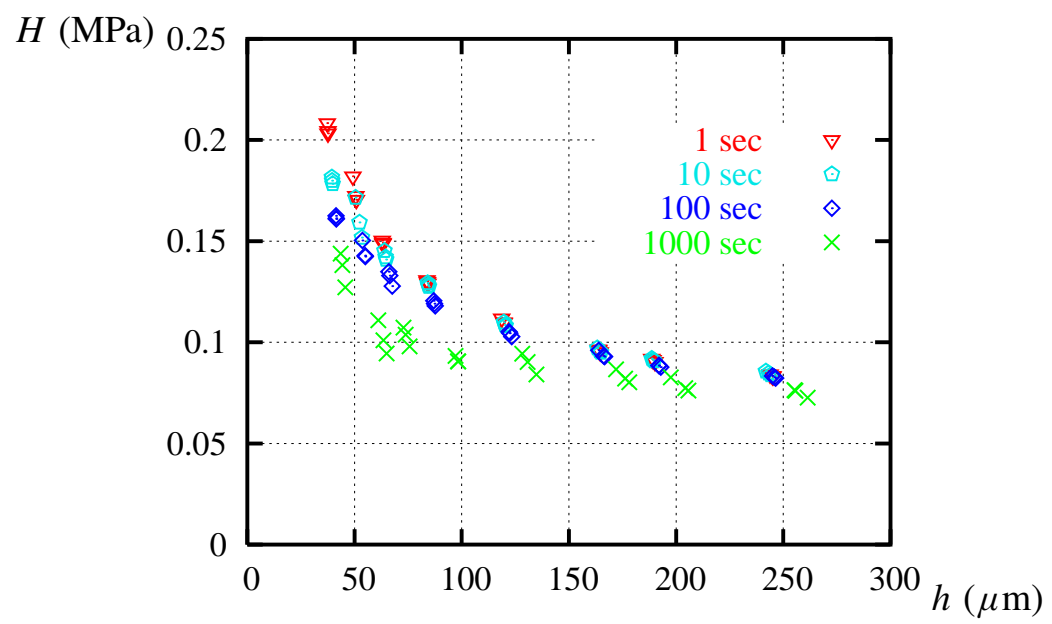

Figure 4. Universal hardness $H$ versus indentation depth $h$ for various loading times. 
versus $h$ for different loading times; here $F$ denotes the force applied by the indenter and $A_{\mathrm{s}}=26.44 h^{2}$ is the nominal contact area of the indenter penetrating beyond the zero-point of contact, $h$, described above [ISO 14577-1 2002].

The universal or Martens hardness was chosen because the determination of the indentation hardness has been developed for metallic materials where the elastic deformation is small compared to the inelastic deformation [Oliver and Pharr 1992], and is therefore questionable for a highly elastic material such as silicone rubber; while the universal hardness can be applied to all materials [ISO 14577-1 2002]. If size effects are not present both the universal hardness and the indentation hardness are independent of $h$. As can be seen in Figure 4, however, a significant increase in the universal hardness, $H$, has been determined for small indentation depths below about 100 microns. The figure also shows that with longer indentation times and corresponding smaller strain rates $H$ decreases. Such increased hardness at lower loading times has also been observed by others [Shen et al. 2004], and is consistent with the increased dissipation at lower loading times shown in Figure 3. Assuming that the deformation in the considered silicone rubber is of viscoelastic nature the data in Figure 3 would indicate that the viscosity and rate dependence are increasing with decreasing $h$.

\section{Analysis and evaluation of experimental data}

In the above described experiments we observed significant indentation size effects at indentation depths between 30 and 300 microns and, with decreasing loading times, the dissipation and the indentation size effect increased. While the dissipation increases with decreasing $h$ the elastic indentation work $W_{\mathrm{I}}^{\mathrm{e}}$ is large compared to the dissipation $W_{\mathrm{I}}^{\mathrm{v}}$ in all indentations performed here. For an analysis of the $h$-dependent universal hardness, the rate-independent hardness model suggested in [Han and Nikolov 2007] may therefore be considered as an approximation, where rate effects have been neglected.

It is well established that the deformation of liquid crystal polymers can be described by the Frank energy and the related rotational gradients [de Gennes and Prost 1993; Stewart 2004] which originate from molecular interactions. While the here-considered filled silicone rubber is not a liquid crystal polymer it is here assumed that for glassy polymers the molecular interactions related to the Frank energy still play an important role in the deformation process at small scales because the lack of nematic order is due to topological defects like cross-links and entanglements rather than to the absence of sufficiently strong molecular interactions. Following this assumption, that the Frank energy is also relevant in amorphous polymers, a Frank elasticity type rotation gradient energy was suggested by Nikolov et al. [2007] to account for the observed elastic size effects in the bending of epoxy microbeams [Lam et al. 2003; McFarland and Colton 2005]. A corresponding extension for elastoplastic materials and a related hardness model were deduced in [Han and Nikolov 2007] by including a Frank energy related indentation work term $W_{\mathrm{I}}^{\mathrm{F}}$ in the total indentation work. For viscoelastic materials this extension of (2) can be given by $W_{\mathrm{I}}=W_{\mathrm{I} \varepsilon}^{\mathrm{e}}+W_{\mathrm{I} \varepsilon}^{\mathrm{v}}+W_{\mathrm{I}}^{\mathrm{F}}$, where the subscript $\varepsilon$ has been added to indicate the local nature of these indentation work components. The Frank energy density in its one-parameter form $W^{\mathrm{F}}=\frac{1}{2} K\langle\nabla \boldsymbol{n}: \nabla \boldsymbol{n}\rangle=\frac{1}{2} K\left\langle n_{i, j} n_{i, j}\right\rangle$ is a quadratic function in the gradients of the orientations of the more rigid (nematic) parts of the polymer chain $\boldsymbol{n}$ (averaged over all directions, which is indicated by \langle\rangle$)$ and is also directly proportional to the Frank constant $K$, which depends on the molecular structure and properties of the polymer [Liu and Fredrickson 1993]. The change in the Frank energy density from 
the initial configuration $\boldsymbol{n}^{0}$ to the current configuration $\boldsymbol{n}$ can then be expressed by

$$
\Delta W^{\mathrm{F}}=\frac{K}{2}\left(\left\langle n_{i, j} n_{i, j}\right\rangle-\left\langle n_{i, j}^{0} n_{i, j}^{0}\right\rangle\right) .
$$

This expression is not a practical description in deformation related terms. Assuming, firstly, a decoupling of the local work densities $W_{\varepsilon}^{\mathrm{e}}, W_{\varepsilon}^{\mathrm{v}}$, and the Frank energy and that, secondly, a Frank energy related deformation potential is zero in the undeformed state and increases with deformation, Nikolov et al. [2007] motivated a Frank energy type deformation potential $\Delta \tilde{W}^{\mathrm{F}}$

$$
\Delta \tilde{W}^{\mathrm{F}}=\frac{\tilde{K}}{3} \chi_{i j}^{\mathrm{S}} \chi_{i j}^{\mathrm{S}}
$$

where $\chi_{i j}^{\mathrm{S}}=\frac{1}{2}\left(\chi_{i j}+\chi_{j j}\right)$ and $\chi_{i j}=\frac{1}{2} e_{i n m} u_{m, j n}$ represent gradients in the rotations. The displacement field is $u_{m}$ and the permutation symbol is $e_{j n m}$. The tilde is introduced in (5) to differentiate it from the Frank energy, (4), due to the applied assumptions and approximations. In spite of the modifications of the Frank energy, (4), the $K$-related constant $\tilde{K}$ in (5) should be of the same order.

With $\Delta \tilde{W}^{\mathrm{F}}$, the total deformation work density is suggested as

$$
W=W_{\varepsilon}^{\mathrm{e}}+W_{\varepsilon}^{\mathrm{v}}+\Delta \tilde{W}^{\mathrm{F}},
$$

where $W_{\varepsilon}^{\mathrm{v}}$ corresponds to the indentation work component $W_{\mathrm{I} \varepsilon}^{\mathrm{v}}$ and is described in local stress and strain related terms.

To a first order approximation the magnitude of the rotation gradient $\chi^{\mathrm{S}}$ averaged over the area under the indenter increases with decreasing $h$ as the distances are proportional to $h$. Consequently for large indentation depths $h$ the nonlocal work $W_{\mathrm{I}}^{\mathrm{F}}$ should become small relative to $W_{\mathrm{I} \varepsilon}^{\mathrm{e}}+W_{\mathrm{I} \varepsilon}^{\mathrm{v}}$. With decreasing $h$, however, the energy $W_{\mathrm{I}}^{\mathrm{F}}$ to be exerted during indentation will increase relative to $W_{\mathrm{I} \varepsilon}^{\mathrm{e}}+W_{\mathrm{I} \varepsilon}^{\mathrm{v}}$ resulting in higher applied forces and corresponding higher hardness values (see [Han and Nikolov 2007] for more details).

Based on the additional indentation work $W_{\mathrm{I}}^{\mathrm{F}}$ associated with the deformation work density $\Delta \tilde{W}^{\mathrm{F}}$, an approximate hardness model,

$$
H=H_{0}\left(1+\frac{c_{\ell}}{h}\right) \text {, }
$$

was deduced in [Han and Nikolov 2007]. It involves a length scale parameter $c_{\ell}$ and a macroscopic hardness $H_{0}$, which also represents the lower limit of $H$. This model was developed by neglecting rate effects and assuming that the essential characteristics will not change with the loading time. A more general model discussed in the same paper is $H=H_{0}\left(1+\left(c_{\ell} / h\right)^{\gamma}\right)$, with a fitting parameter accounting for the assumptions applied in its deduction. For $\gamma=1 / 2$ this is identical to the model suggested in [Chong and Lam 1999]. For a short range $h$ the differences from using (7), that is, $\gamma=1$, and $\gamma=1 / 2$ are small, but they increase with the range of $h$.

Equation (7) describes the $h-H$ relation fairly well for elastoplastically deforming epoxy, polycarbonate, and polystyrene for which experimental data of up to 10 microns were reported in the literature [Chong and Lam 1999]. For small indentation depths (under $50 \mathrm{~nm}$ or so), effects from surface roughness, indenter tip curvature, and surface effects may be present, as discussed in the introduction. Equation (7) does not take into account these effects and consequently does not represent the size-dependent hardness below about $50 \mathrm{~nm}$ indentation depth. 


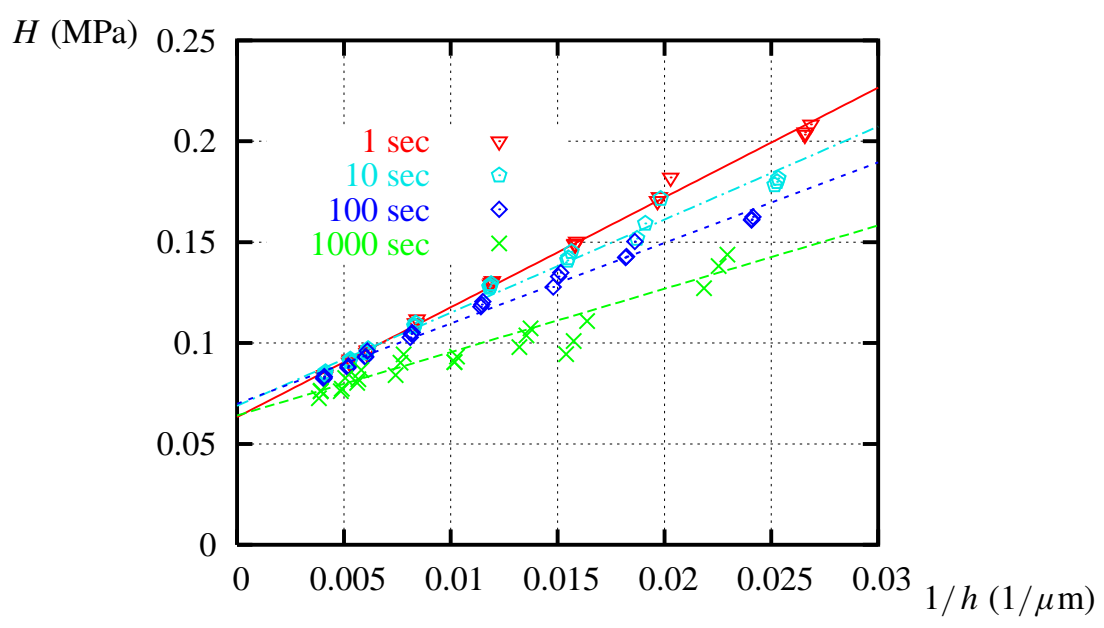

Figure 5. Universal hardness $H$ versus inverse indentation depth $1 / h$.

The dissipation is rather small in the indentation of our silicone material (see Figure 3) and therefore one could assume that the above approach should be a good approximation. In fact, (7) was also found to predict the $h-H$ relation quite well for different loading times, as can be seen in Figure 5 where $1 / h$ versus $H$ is shown. The figure also contains linear curves in $1 / h$ obtained from least square fits of the parameters of (7), that is, $H_{0}$ and $c_{\ell}$. As can be seen from these figures the deviation of the experimental data from these linear curves in $1 / h$ is rather small. As the differences in $H$ at small loading times increase with decreasing $h$ it can be concluded that rate effects are pronounced and thus more important at small indentations, while at large indentation depth rate effects are less important.

The numerical values of these least square fits are given in Table 1, where the macroscopic hardness $H_{0}$ varies little with the loading time, while there are quite significant increases of $c_{\ell}$ with decreasing $t_{\mathrm{R}}$. This can also be seen in Figure 5 where the fitted lines for different $t_{\mathrm{R}}$ values more or less converge to a common $H_{0}$ at $1 / h=0$. This would imply that there is little rate dependence in the macroscopic hardness $H_{0}$ which in turn also implies that there is little rate dependence in the macroscopic deformation (that is, the deformation with or without small rotation gradients).

\begin{tabular}{|l|cccc|}
\hline Loading time $t_{\mathrm{R}}$ & $1 \mathrm{~s}$ & $10 \mathrm{~s}$ & $100 \mathrm{~s}$ & $1000 \mathrm{~s}$ \\
$H_{0}(\mathrm{MPa})$ & 0.0633 & 0.0689 & 0.0698 & 0.0633 \\
$c_{\ell}(\mu \mathrm{m})$ & 85.988 & 66.972 & 57.192 & 47.442 \\
\hline
\end{tabular}

Table 1. Least square fits for $H_{0}$ and $c_{\ell}$.

\section{Discussion}

Adhesion forces may be of importance at indentation depths below the micron range, as reported in [Carrillo et al. 2005] where a spherical indenter tip was used at indentation depths of about $500 \mathrm{~nm}$. With a cone shaped indenter tip and in the indentation depth range considered herein, between 30 and 300 microns, the work of adhesion should, however, be less significant, or negligible compared to the 
Macroscopic/local component

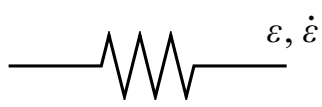

Microscopic/nonlocal component

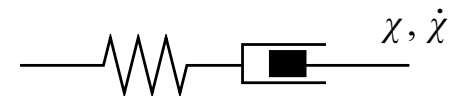

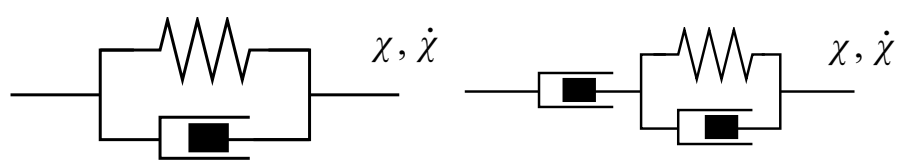

Figure 6. Rheological representation at the macro and micro levels (including the Maxwell, Kelvin-Voigt, and combined models).

indentation work components indicated in Figure 1. In this respect it is worth noting that in a numerical analysis friction effects [Mata and Alcalá 2004] could not be related to the indentation size effect. Similarly adhesion forces should also scale with the contact area and should therefore be affine with the indentation depth. These are therefore unlikely to be the cause of the indentation size effect. Using conical indenter tips the effect of the indenter tip curvature is of more relevance at submicron indentation depths [Qu et al. 2004] than the here-considered range of 30-300 microns. While rate effects were not investigated in [Lim and Chaudhri 2006] it was found that for a silicone rubber with a higher cross-link density elevated hardnesses were also observed for small indentation depths.

As opposed to [Lim and Chaudhri 2006], the silicone rubber material used in this work is not a pure polydimethylsiloxane as described in Section 2. The silicone rubber used here is more of a polymer blend, and that has not been taken into consideration in the analysis and evaluation given in Section 4. There is also a lack of clarity about the length scale of the second phases that may be present due to the fumed silica reinforced vinyl-terminated polydimethylsiloxane and the divinyltetramethyldisiloxane complex in xylene used in the fabrication process of the samples used in this work. We believe however that the issue of quantification/characterization of microscopic phases and corresponding possibly present anisotropy would be quite complicated and beyond the scope and intent of this work. Given the fact, however, that such an indentation size effect in a similar indentation depth range has also been found in [Lim and Chaudhri 2006] for highly cross-linked polydimethylsiloxane, this may indicate that the notion suggested in Section 4 is nevertheless valid for the silicone rubber used in this work as well as the highly cross-linked polydimethylsiloxane of [Lim and Chaudhri 2006]. The possible differences between the polydimethylsiloxane of [Lim and Chaudhri 2006] and the polymer blend used here fabricated with fumed silica reinforced vinyl terminated polydimethylsiloxane remains an open question which is not considered in this article.

As the depth-dependent hardness cannot be related to $W_{\mathrm{I} \varepsilon}^{\mathrm{e}}$ and $W_{\mathrm{I} \varepsilon}^{\mathrm{v}}$, the small changes in $H_{0}$ at different $t_{\mathrm{R}}$ values indicate that the rate effects are mostly related to the rotational gradient-dependent $W_{\mathrm{I}}^{\mathrm{F}}$. That would necessitate a reformulation of $\Delta \tilde{W}^{\mathrm{F}}$, as it should be rate-dependent and not purely elastic, as described in (5).

The viscoelastic nature of the Frank elasticity per se has already been investigated for liquid crystal polymers both in theory and experiment [Jamieson et al. 1996]. Possible combined micro-macro rheological models are suggested in Figure 6, where the macroscopic model is not rate-sensitive while the microscopic model includes damping terms dependent on $\dot{\chi}$. Note that because the macroscopic model is an elastic solid the overall local/nonlocal model would remain rheologically a (viscoelastic) solid even 
if the nonlocal rheological component on its own would be a fluid. A corresponding constitutive material formulation in $\chi^{\mathrm{S}}$ and its rate would necessitate a decomposition of $\chi^{\mathrm{S}}$ into elastic and viscous parts. The constitutive equations and boundary conditions of such a material model would be quite complex. A phenomenological theory for elastoplastic materials with such a decomposition of a strain gradient tensor into elastic and plastic parts has been, for instance, suggested in [Chen and Wang 2002].

\section{Conclusions}

The rate dependence of indentation size effects in silicone rubber has been studied in the range of 30300 microns and 1-1000 seconds. From the experimental results we can draw the following conclusions:

- The dissipation and hardness $H$ increase with decreasing indentation depth $h$ and with decreasing loading time.

- The rate dependence increases with decreasing indentation depth for the investigated silicone rubber. Therefore rate effects are very significant and important at small indentation depths of below 100 microns.

- The rate-independent hardness model $H=H_{0}\left(1+\frac{c_{\ell}}{h}\right)$ describes the hardness indentation depth relation well for a constant loading time.

- In this model the length scale parameter $c_{\ell}$ decreases with loading time, while $H_{0}$ as a projected value of the macroscopic hardness is relatively unaffected by the loading time. Consequently rate effects are mostly associated with nonlocal deformation.

Acknowledgments. The support of this work by the North Dakota EPSCoR Program (EPS-0447679) and the National Science Foundation through the CAREER program (Grant CMMI 0846692) is appreciated. The authors would also like to acknowledge the assistance of Eon-Chul Song in producing the silicone samples and the valuable comments and discussions with Dr. Hosup Jung.

\section{References}

[Briscoe et al. 1998] B. J. Briscoe, L. Fiori, and E. Pelillo, "Nano-indentation of polymeric surfaces", J. Phys. D Appl. Phys. 31:19 (1998), 2395-2405.

[Carrillo et al. 2005] F. Carrillo, S. Gupta, M. Balooch, S. J. Marshall, G. W. Marshall, L. Pruitt, and C. M. Puttlitz, "Nanoindentation of polydimethylsiloxane elastomers: effect of crosslinking, work of adhesion, and fluid environment on elastic modulus", J. Mater. Res. 20:10 (2005), 2820-2830.

[Chen and Wang 2002] S. H. Chen and T. C. Wang, "A new deformation theory with strain gradient effects", Int. J. Plast. 18:8 (2002), 971-995.

[Chong and Lam 1999] A. C. M. Chong and D. C. C. Lam, "Strain gradient plasticity effect in indentation hardness of polymers", J. Mater. Res. 14:10 (1999), 4103-4110.

[Dao et al. 2001] M. Dao, N. Chollacoop, K. J. Van Vliet, T. A. Venkatesh, and S. Suresh, "Computational modeling of the forward and reverse problems in instrumented sharp indentation", Acta Mater. 49:19 (2001), 3899-3918.

[Ebenstein and Wahl 2006] D. M. Ebenstein and K. J. Wahl, "A comparison of JKR-based methods to analyze quasi-static and dynamic indentation force curves", J. Colloid Interface Sci. 298:2 (2006), 652-662.

[de Gennes and Prost 1993] P. G. de Gennes and J. Prost, The physics of liquid crystals, 2nd ed., International Series of Monographs on Physics 83, Oxford University Press, New York, 1993.

[Gudmundson 2006] P. Gudmundson, "Modelling of length scale effects in viscoelastic materials", Eur. J. Mech. A Solids 25:3 (2006), 379-388. 
[Han 2010] C.-S. Han, "Influence of the molecular structure on indentation size effect in polymers", Mater. Sci. Eng. A 527:3 (2010), 619-624.

[Han and Nikolov 2007] C.-S. Han and S. Nikolov, "Indentation size effects of polymers and related rotation gradients", $J$. Mater. Res. 22:6 (2007), 1662-1672.

[Han et al. 2006] C.-S. Han, A. Hartmaier, H. Gao, and Y. Huang, "Discrete dislocation dynamics simulations of surface induced size effects in plasticity”, Mater. Sci. Eng. A 415:1-2 (2006), 225-233.

[Han et al. 2007a] C.-S. Han, A. Ma, F. Roters, and D. Raabe, "A finite element approach with patch projection for strain gradient plasticity formulations", Int. J. Plast. 23:4 (2007), 690-710.

[Han et al. 2007b] C.-S. Han, C. Zhang, J. Wang, J. Kim, and S.-B. Choi, "Finite element analysis of thickness dependent debonding forces of elastomer coatings", J. Adhesion 83:6 (2007), 535-551.

[ISO 14577-1 2002] ISO 14577-1, "Metallic materials - instrumented indentation test for hardness and materials parameters, 1: Test method", 2002, Available at http://tinyurl.com/ISO-14577-1-2002.

[Jamieson et al. 1996] A. M. Jamieson, D. Gu, F. L. Chen, and S. Smith, "Viscoelastic behavior of nematic monodomains containing liquid crystal polymers", Prog. Polym. Sci. 21:5 (1996), 981-1033.

[Lam et al. 2003] D. C. C. Lam, F. Yang, A. C. M. Chong, J. Wang, and P. Tong, "Experiments and theory in strain gradient elasticity", J. Mech. Phys. Solids 51:8 (2003), 1477-1508.

[Larsson and Carlsson 1998] P.-L. Larsson and S. Carlsson, “On microindentation of viscoelastic polymers”, Polym. Test. 17:1 (1998), 49-75.

[Lemaitre and Chaboche 1990] J. Lemaitre and J.-L. Chaboche, Mechanics of solid materials, Cambridge University Press, Cambridge, 1990.

[Li and Bhushan 2000] X. Li and B. Bhushan, "Continuous stiffness measurement and creep behavior of composite magnetic tapes", Thin Solid Films 377-378 (2000), 401-406.

[Lim and Chaudhri 2006] Y. Y. Lim and M. M. Chaudhri, "Indentation of elastic solids with a rigid Vickers pyramidal indenter", Mech. Mater. 38:12 (2006), 1213-1228.

[Liu and Fredrickson 1993] A. J. Liu and G. H. Fredrickson, "Free energy functionals for semiflexible polymer solutions and blends", Macromolecules 26:11 (1993), 2817-2824.

[Ma and Clarke 1995] Q. Ma and D. R. Clarke, "Size dependent hardness of silver single crystals”, J. Mater. Res. 10:4 (1995), 853-863.

[Mata and Alcalá 2004] M. Mata and J. Alcalá, “The role of friction on sharp indentation”, J. Mech. Phys. Solids 52:1 (2004), $145-165$.

[McFarland and Colton 2005] A. W. McFarland and J. S. Colton, "Role of material microstructure in plate stiffness with relevance to microcantilever sensors", J. Micromech. Microeng. 15:5 (2005), 1060-1067.

[Nikolov et al. 2007] S. Nikolov, C.-S. Han, and D. Raabe, "On the origin of size effects in small-strain elasticity of solid polymers", Int. J. Solids Struct. 44:5 (2007), 1582-1592. Corrigendum: Int. J. Solids Struct. 44:22-23 (2007), 7713.

[Nix and Gao 1998] W. D. Nix and H. Gao, "Indentation size effects in crystalline materials: a law for strain gradient plasticity", J. Mech. Phys. Solids 46:3 (1998), 411-425.

[Oliver and Pharr 1992] W. C. Oliver and G. M. Pharr, "An improved technique for determining hardness and elastic modulus using load and displacement sensing indentation experiments", J. Mater. Res. 7:6 (1992), 1564-1583.

[Oyen 2006] M. L. Oyen, "Analytical techniques for indentation of viscoelastic materials", Philos. Mag. 86:33-35 (2006), $5625-5641$.

[Qu et al. 2004] S. Qu, Y. Huang, W. D. Nix, H. Jiang, F. Zhang, and K. C. Hwang, "Indenter tip radius effect on the Nix-Gao relation in micro- and nanoindentation hardness experiments", J. Mater. Res. 19:11 (2004), 3423-3434.

[Sargent and Ashby 1992] P. M. Sargent and M. F. Ashby, "Indentation creep”, Mater. Sci. Technol. 8:7 (1992), 594-601.

[Shen et al. 2004] L. Shen, I. Y. Phang, T. Liu, and K. Zeng, "Nanoindentation and morphological studies on nylon 66/organoclay nanocomposites, II: Effect on strain rate", Polymer 45:24 (2004), 8221-8229.

[Stewart 2004] I. W. Stewart, The static and dynamic continuum theory of liquid crystals, Taylor and Francis, London, 2004. 
[Tan et al. 2007] J. Tan, Y. J. Chao, X. Li, and J. W. Van Zee, "Degradation of silicone rubber under compression in a simulated PEM fuel cell environment", J. Power Sources 172:2 (2007), 782-789.

[Tatiraju et al. 2008] R. V. S. Tatiraju, C.-S. Han, and S. Nikolov, "Size dependent hardness of polyamide/imide", Open Mech. J. 2 (2008), 89-92.

[Tjernlund et al. 2004] J. A. Tjernlund, E. K. Gamstedt, and Z.-H. Xu, "Influence of molecular weight on strain-gradient yielding in polystyrene", Polym. Eng. Sci. 44:10 (2004), 1987-1997.

[Tweedie and Van Vliet 2006] C. A. Tweedie and K. J. Van Vliet, "On the indentation recovery and fleeting hardness of polymers", J. Mater. Res. 21:12 (2006), 3029-3036.

[Tweedie et al. 2007] C. A. Tweedie, G. Constantinides, K. E. Lehman, D. J. Brill, G. S. Blackman, and K. J. Van Vliet, "Enhanced stiffness of amorphous polymer surfaces under confinement of localized contact loads", Adv. Mater. 19:18 (2007), 2540-2546.

[Xu and Zhang 2004] W.-H. Xu and T.-Y. Zhang, "Surface effect for different types of materials in nanoindentation”, Key Eng. Mat. 261-263 (2004), 1587-1592.

[Yang et al. 2002] F. Yang, A. C. M. Chong, D. C. C. Lam, and P. Tong, "Couple stress based strain gradient theory for elasticity", Int. J. Solids Struct. 39:10 (2002), 2731-2743.

[Zhang and Xu 2002] T.-Y. Zhang and W.-H. Xu, "Surface effects on nanoindentation", J. Mater. Res. 17:7 (2002), 1715-1720.

[Zhang et al. 2004] T.-Y. Zhang, W.-H. Xu, and M.-H. Zhao, "The role of plastic deformation of rough surfaces in the sizedependent hardness", Acta Mater. 52:1 (2004), 57-68.

Received 24 Jan 2009. Revised 13 Aug 2009. Accepted 19 Aug 2009.

RAMANJANEYULU V. S. TATIRAJU: ramanjaneyulu.tatiraju@ndsu.edu

North Dakota State University, Department of Civil Engineering, PO Box 6050, Fargo, ND 58108, United States

CHUNG-SoUK HAN: chung-souk. han@ndsu.edu

North Dakota State University, Department of Civil Engineering, PO Box 6050, Fargo, ND 58108, United States and

University of Wyoming, Department of Mechanical Engineering (Dept. \#3295), 1000 E. University Avenue, Laramie, WY 82071, United States 


\title{
JOURNAL OF MECHANICS OF MATERIALS AND STRUCTURES
}

\author{
http://www.jomms.org
}

\author{
Founded by Charles R. Steele and Marie-Louise Steele

\section{EDITORS} \\ Charles R. STEele \\ DAVIDE BIGONI \\ IWONA JASIUK \\ YASUHIDE SHINDO \\ Stanford University, U.S.A. \\ University of Trento, Italy \\ University of Illinois at Urbana-Champaign, U.S.A. \\ Tohoku University, Japan
}

\section{EDITORIAL BOARD}

H. D. BUI École Polytechnique, France

J. P. CARTER University of Sydney, Australia

R. M. Christensen Stanford University, U.S.A.

G. M. L. GLADWELL University of Waterloo, Canada

D. H. HodGES Georgia Institute of Technology, U.S.A.

J. HUTCHINSON Harvard University, U.S.A.

C. HwU National Cheng Kung University, R.O. China

B. L. KariHaloo University of Wales, U.K.

Y. Y. KIM Seoul National University, Republic of Korea

Z. Mroz Academy of Science, Poland

D. PAMPlonA Universidade Católica do Rio de Janeiro, Brazil

M. B. RUBIN Technion, Haifa, Israel

A. N. SHUPIKov Ukrainian Academy of Sciences, Ukraine

T. TARNAI University Budapest, Hungary

F. Y. M. WAN University of California, Irvine, U.S.A.

P. WRIGGERS Universität Hannover, Germany

W. YANG Tsinghua University, P.R. China

F. ZIEGLER Technische Universität Wien, Austria

\section{PRODUCTION}

\section{Paulo Ney de Souza Production Manager \\ SheIla Newbery Senior Production Editor \\ SILVIO LEVY Scientific Editor}

See inside back cover or http://www.jomms.org for submission guidelines.

JoMMS (ISSN 1559-3959) is published in 10 issues a year. The subscription price for 2010 is US $\$ 500 /$ year for the electronic version, and \$660/year (+\$60 shipping outside the US) for print and electronic. Subscriptions, requests for back issues, and changes of address should be sent to Mathematical Sciences Publishers, Department of Mathematics, University of California, Berkeley, CA 94720-3840.

JoMMS peer-review and production is managed by EditFLOW ${ }^{\mathrm{TM}}$ from Mathematical Sciences Publishers.

PUBLISHED BY

mathematical sciences publishers

http://www.mathscipub.org

A NON-PROFIT CORPORATION

Typeset in LATEX

CCopyright 2010. Journal of Mechanics of Materials and Structures. All rights reserved. 


\title{
Journal of Mechanics of Materials and Structures
}

\author{
Volume 5, No. 2 February 2010
}

A critical analysis of interface constitutive models for the simulation of delamination in composites and failure of adhesive bonds

Anton Matzenmiller, Sebastian Gerlach and Mark Fiolka

185

Computational studies of collagen fibril biominerals using a virtual internal bond

model with extrinsic length scale

Ganesh Thiagarajan and Kavita Deshmukh

The simulation of stochastically excited viscoelastic systems and their stability

VADIM D. POTAPOV

Fundamental solutions for an inhomogeneous cross-anisotropic material due to horizontal and vertical plane strain line loads

Cheng-Der Wang, Jia-YAN Hou and WeI-Jer WANG

Mechanical and fracture analysis of welded pearlitic rail steels

Aldinton Allie, Heshmat A. Aglan and Mahmood Fateh

Rate dependence of indentation size effects in filled silicone rubber

Ramanjaneyulu V. S. TATIRAJU and ChUng-SOUK HAN

A novel application of a laser Doppler vibrometer in a hèalth monitoring system

DAVOOD REZAEI and FARID TAHERI

Energy absorption of a helicoidal bistable structure

Seubpong Leelavanichkul, Andrej Cherkaev, Daniel O. Adams

and FLORIAN SOLZBACHER

Decay properties of solutions of a Mindlin-type plate model for rhombic systems

Francesca Passarella, Vincenzo Tibullo and VitTorio Zampoli

A consistent refinement of first-order shear deformation theory for laminated composite and sandwich plates using improved zigzag kinematics Alexander Tessler, Marco Di Sciuva and Marco Gherlone 